\title{
SOME NEW $Z$-EIGENVALUE LOCALIZATION SETS FOR TENSORS AND THEIR APPLICATIONS
}

\author{
ZHENGGE HUANG, LIGONG WANG, ZHONG XU, AND JINGJING CUI
}

\begin{abstract}
In this paper some new $Z$-eigenvalue localization sets for general tensors are established, which are proved to be tighter than those newly derived by Wang et al. [Discrete Contin. Dyn. Syst. Ser. B, 22 (2017), 187-198]. Also, some relationships between the $Z$-eigenvalue inclusion sets presented by Wang et al. and the new $Z$-eigenvalue localization sets for tensors are given. Besides, we discuss the effects of orthonormal transformations for the proposed sets. As applications of the proposed sets, some improved upper bounds for the $Z$-spectral radius of weakly symmetric nonnegative tensors are given. Numerical examples are also given to verify the advantages of our proposed results over some known ones.
\end{abstract}

\section{INTRODUCTION}

For a positive integer $n$, let $N=\{1,2, \ldots, n\}$ and $\mathbb{C}(\mathbb{R})$ denote the set of all complex (real) numbers. $\mathcal{A} \in \mathbb{C}^{[m, n]}\left(\mathbb{R}^{[m, n]}\right)$ denotes the order $m$ dimension $n$ tensor with $n^{m}$ complex (real) entries, where $i_{j} \in N$ for $j=1,2, \ldots, m$. A real tensor $\mathcal{A}=\left(a_{i_{1} \ldots i_{m}}\right)$ is called symmetric [29, 42, 16, 17, 20, 21] if

$$
a_{i_{1} \ldots i_{m}}=a_{\pi\left(i_{1} \ldots i_{m}\right)}, \quad \forall \pi \in \Pi_{m},
$$

where $\Pi_{m}$ is the permutation group of $m$ indices. Furthermore, a real tensor of order $m$ dimension $n$ is called the unit tensor [18], denoted by $\mathcal{I}$, if its entries are $\delta_{i_{1} \ldots i_{m}}$ for $i_{1}, \ldots, i_{m} \in N$, where

$$
\delta_{i_{1} \ldots i_{m}}= \begin{cases}1, & \text { if } i_{1}=\cdots=i_{m} \\ 0, & \text { otherwise }\end{cases}
$$

2010 Mathematics Subject Classification. 15A18, 15A42, 15A69.

Key words and phrases. Z-eigenvalue localization set; Weakly symmetric nonnegative tensors; Largest $Z$-eigenvalue; Spectral radius.

This research was supported by the National Natural Science Foundation of China (No. 11871398), the Natural Science Basic Research Plan in Shaanxi Province of China (Program No. 2018JM1032) and the Innovation Foundation for Doctor Dissertation of Northwestern Polytechnical University (No. CX201628). 
Throughout this paper, for $\mathcal{A}=\left(a_{i_{1} \ldots i_{m}}\right) \in \mathbb{R}^{[m, n]}$ and $i, j \in N, j \neq i$, the following notations are utilized repeatedly in our proofs.

$$
\begin{aligned}
R_{i}(\mathcal{A}) & =\sum_{i_{2}, \ldots, i_{m}=1}^{n}\left|a_{i i_{2} \ldots i_{m}}\right|, \\
P_{i}^{i}(\mathcal{A}) & =\sum_{\substack{i_{2}, \ldots, i_{m} \in N, i \notin\left\{i_{2}, \ldots, i_{m}\right\}}}\left|a_{i i_{2} \ldots i_{m}}\right|, \\
P_{j}^{i}(\mathcal{A}) & =\sum_{\substack{i_{2}, \ldots, i_{m} \in N, i \notin\left\{i_{2}, \ldots, i_{m}\right\}}}\left|a_{j i_{2} \ldots i_{m}}\right| .
\end{aligned}
$$

For each vector $x=\left(x_{1}, \ldots, x_{n}\right)^{T}$ of dimension $n$, real or complex, we define the following vector of dimension $n$ [24]:

$$
\mathcal{A} x^{m-1}=\left(\sum_{i_{2}, \ldots, i_{m}=1}^{n} a_{i i_{2} \ldots i_{m}} x_{i_{2}} \cdots x_{i_{m}}\right)_{1 \leq i \leq n} .
$$

The definitions of $H$-eigenvalue and $Z$-eigenvalue of tensors were first introduced and studied by Qi and Lim [29, 27].

Definition $1.1([29])$. A pair $(\lambda, x) \in \mathbb{C} \times\left(\mathbb{C}^{n} /\{0\}\right)$ is called an eigenpair of $\mathcal{A}$ if

$$
\mathcal{A} x^{m-1}=\lambda x^{[m-1]},
$$

where $x^{[m-1]}=\left(x_{1}^{m-1}, x_{2}^{m-1}, \ldots, x_{n}^{m-1}\right)^{T}$. Here $x^{T}$ denotes the transpose of $x$. Furthermore, we call $(\lambda, x)$ an $H$-eigenpair, if both $\lambda$ and $x$ are real.

This definition was introduced by Qi in [29] where he assumed that $\mathcal{A}=\left(a_{i_{1} \ldots i_{m}}\right) \in$ $\mathbb{R}^{[m, n]}$ is symmetric and $m$ is even. Independently, in [27], Lim gave such a definition but restricted $x$ to be a real vector and $\lambda$ to be a real number. It is noteworthy that the definition of eigenvalues of tensors proposed by Lim in [27] is different from Definition 1.1 in the case of odd order as the right hand side of 1.1 is taken absolute value.

Definition $1.2([29,27])$. A pair $(\lambda, x) \in \mathbb{C} \times\left(\mathbb{C}^{n} \backslash\{0\}\right)$ is called an E-eigenpair of $\mathcal{A}$, if they satisfy the equation

$$
\left\{\begin{array}{l}
\mathcal{A} x^{m-1}=\lambda x \\
x^{T} x=1
\end{array}\right.
$$

We call $(\lambda, x)$ a $Z$-eigenpair, if both $\lambda$ and $x$ are real.

Definition $1.3([29,39,11,24])$. We define $\sigma(\mathcal{A})$ the $Z$-spectrum of $\mathcal{A}$ by the set of all $Z$-eigenvalues of $\mathcal{A}$. Assume $\sigma(\mathcal{A}) \neq \emptyset$. Then the $Z$-spectral radius of $\mathcal{A}$ is defined by

$$
\rho(\mathcal{A})=\max \{|\lambda|: \lambda \in \sigma(\mathcal{A})\}
$$


Due to the fact that both $H$-eigenvalue and $Z$-eigenvalue problems of tensors have become important topics in numerical multilinear algebra, and they have wide applications in magnetic resonance imaging [34, higher order Markov chains 28], spectral hypergraph theory [7] and so forth, there has been a surge of interest in spectral theory of tensors recently $[29,31,30,3,4,4,42,40,10,11,39,36,26,24$, 8, 16, 9].

Definition $1.4([3,42,40,26,30,12])$. A tensor $\mathcal{A}$ is called nonnegative (positive), denoted by $\mathcal{A} \geq 0(\mathcal{A}>0)$, if any entry $a_{i_{1} i_{2} \ldots i_{m}} \geq 0\left(a_{i_{1} i_{2} \ldots i_{m}}>0\right.$, respectively).

Definition $1.5([5,11,24,37])$. A tensor $\mathcal{A}=\left(a_{i_{1} \cdots i_{m}}\right) \in \mathbb{R}^{[m, n]}$ is called weakly symmetric if the associated homogeneous polynomial

$$
f_{\mathcal{A}}(x)=\mathcal{A} x^{m}=\sum_{i_{1}, i_{2}, \ldots, i_{m}=1}^{n} a_{i_{1} i_{2} \ldots i_{m}} x_{i_{1}} x_{i_{2}} \cdots x_{i_{m}}
$$

satisfies $\nabla f_{\mathcal{A}}(x)=m \mathcal{A} x^{m-1}$.

This concept was first introduced and used by Chang et al. [5] for studying the properties of $Z$-eigenvalue of nonnegative tensors and the authors presented the Perron-Frobenius theorem for the $Z$-eigenvalue of nonnegative tensors, which was later reproved as Lemma 4.7 by Song and Qi in [36], using a different technique.

It is noteworthy that the $Z$-eigenvalue problem plays a significant role in some applications such as high order Markov chains (see e.g. [25, 6]), best rank-one approximations in Statistical Data Analysis (see e.g. [43, 15]). In [5, 33, 23], the authors focused on investigating the effective algorithms for finding $Z$-eigenpair of tensors.

However, as mentioned in [37, we can not judge that $Z$-eigenvalues generated by the aforementioned algorithms are the largest $Z$-eigenvalue in general. To overcome this difficulty, one should study and develop the $Z$-eigenvalue inclusion set which includes all $Z$-eigenvalues of general tensors. For eigenvalue inclusion sets for tensors, many researchers have investigated this topic and many related results have been developed recently, see [29, 16, 17, 18, 2, 20, 21, 22, 14, 11, 13, 38, and references therein. Nevertheless, characterizations of inclusion set for $Z$-eigenvalue are still underdeveloped for general tensors. Based on this fact, Wang et al. [37] proposed some $Z$-eigenvalue inclusion sets which capture all $Z$-eigenvalues of general tensors. Very recently, several new $Z$-eigenvalue inclusion sets for tensors have been put forward, see [41, 35] and the references therein. To capture all $Z$-eigenvalues of a tensor more precisely than some newly proposed results, in this paper we derive some new $Z$-eigenvalue localization sets for general tensors, which are proved to be sharper than the ones in [37].

In [29], Qi extended the well-known Geršgorin eigenvalue localization set of matrices to real symmetric tensors of higher order, and this result can be easily generalized to general tensors [42, 20]. However, Example 1 in [37] shows that the Geršgorin eigenvalue localization set can not be generalized to $Z$-eigenvalues of a general tensor and the authors in [37] established the Geršgorin $Z$-eigenvalue inclusion theorem for general tensors as follows. 
Lemma 1.6 (37]). Let $\mathcal{A}=\left(a_{i_{1} \ldots i_{m}}\right) \in \mathbb{C}^{[m, n]}, n \geq 2$. Then all Z-eigenvalues of $\mathcal{A}$ are located in the union of the following sets:

$$
\sigma(\mathcal{A}) \subseteq \mathcal{K}(\mathcal{A})=\bigcup_{i \in N} \mathcal{K}_{i}(\mathcal{A})
$$

where $\mathcal{K}_{i}(\mathcal{A})=\left\{z \in \mathbb{C}:|z| \leq R_{i}(\mathcal{A})\right\}$.

To obtain sharper $Z$-eigenvalue localization sets than $\mathcal{K}(\mathcal{A})$, they also developed other three $Z$-eigenvalue localization sets for general tensors in the following lemmas.

Lemma $1.7(\underline{37}])$. Let $\mathcal{A}=\left(a_{i_{1} \ldots i_{m}}\right) \in \mathbb{C}^{[m, n]}, n \geq 2$. Then all Z-eigenvalues of $\mathcal{A}$ are located in the union of the following sets:

$$
\sigma(\mathcal{A}) \subseteq \mathcal{L}(\mathcal{A})=\bigcup_{i \in N} \bigcap_{j \in N, j \neq i} \mathcal{L}_{i, j}(\mathcal{A})
$$

where $\mathcal{L}_{i, j}(\mathcal{A})=\left\{z \in \mathbb{C}:\left[|z|-\left(R_{i}(\mathcal{A})-\left|a_{i j \ldots j}\right|\right)\right]|z| \leq\left|a_{i j \ldots j}\right| R_{j}(\mathcal{A})\right\}$.

Lemma 1.8 ([37]). Let $\mathcal{A}=\left(a_{i_{1} \ldots i_{m}}\right) \in \mathbb{C}^{[m, n]}$ and $n \geq 2$. Then all $Z$-eigenvalues of $\mathcal{A}$ are located in the union of the following sets:

$$
\sigma(\mathcal{A}) \subseteq \mathcal{M}(\mathcal{A})=\bigcup_{i, j \in N, i \neq j}\left(\mathcal{M}_{i, j}(\mathcal{A}) \bigcup \mathcal{H}_{i, j}(\mathcal{A})\right)
$$

where

$$
\begin{aligned}
\mathcal{M}_{i, j}(\mathcal{A}) & =\left\{z \in \mathbb{C}:\left(|z|-\left[R_{i}(\mathcal{A})-\left|a_{i j \ldots j}\right|\right]\right)\left(|z|-P_{j}^{i}(\mathcal{A})\right) \leq\left|a_{i j \ldots j}\right|\left(R_{j}(\mathcal{A})-P_{j}^{i}(\mathcal{A})\right)\right\}, \\
\mathcal{H}_{i, j}(\mathcal{A}) & \left.=\left\{z \in \mathbb{C}:|z|-\left(R_{i}(\mathcal{A})-\left|a_{i j \ldots j}\right|\right)<0,|z|<P_{j}^{i}(\mathcal{A})\right)\right\} .
\end{aligned}
$$

Lemma $1.9([37])$. Let $\mathcal{A}=\left(a_{i_{1} \ldots i_{m}}\right) \in \mathbb{C}^{[m, n]}$ and $n \geq 2$. Then all $Z$-eigenvalues of $\mathcal{A}$ are located in the union of the following sets:

$$
\sigma(\mathcal{A}) \subseteq \mathcal{N}(\mathcal{A})=\bigcup_{i, j \in N, i \neq j} \mathcal{N}_{i, j}(\mathcal{A})
$$

where $\mathcal{N}_{i, j}(\mathcal{A})=\left\{z \in \mathbb{C}:\left(|z|-\left[R_{i}(\mathcal{A})-P_{i}^{i}(\mathcal{A})\right]\right)|z| \leq P_{i}^{i}(\mathcal{A}) R_{j}(\mathcal{A})\right\}$

Corollaries 3.1-3.3 in [37 showed that the sets in Lemmas 1.7 1.9 are better than the one in Lemma 1.6

Spectral theory of nonnegative tensors has developed rapidly and attracted a lot of researchers in late years. In the meanwhile, they have put forward various bounds to estimate the spectral radius of nonnegative tensors [42, 40, 39, 36, 26], and the upper bounds for the largest $Z$-eigenvalue of tensors are no exception. In [5], Chang et al. proposed upper bounds for the $Z$-spectral radius of nonnegative tensors. On the basis of the relationship between the Gelfand formula and the spectral radius, Song and Qi [36] developed the new upper bounds for the $Z$-spectral radius, which improve the ones in [5]. After that, He and Huang [11] obtained the Ledermannlike upper bound for the largest $Z$-eigenvalue of the weakly symmetric positive tensors. To get the sharper upper bounds for the $Z$-spectral radius, Li et al. 24] gave some new upper bounds and proved that these bounds are tighter than the 
one in [11. Very recently, Wang et al. 37] developed some new upper bounds for the largest $Z$-eigenvalue of weakly symmetric nonnegative tensors by applying the proposed $Z$-eigenvalue inclusion sets in [37]. Numerical results of [37] show that the proposed bounds are tighter than existing bounds of [11, 24, 5]. As mentioned before, estimating the largest $Z$-eigenvalue is of significance. Hence, we continue this research on the $Z$-spectral radius of weakly symmetric nonnegative tensors and propose some new tighter bounds compared with those in [11, 24, 37, 5] on the basis of the new set derived in this paper.

To improve the $Z$-eigenvalue inclusion sets in [37, Theorems 3.1 and 3.3-3.4], some new $Z$-eigenvalue localization sets for general tensors are employed in this paper. They are proved to be more precise than the $Z$-eigenvalue localization sets in Lemmas $1.6,1.8$ and 1.9 Meanwhile, some relationships between the $Z$-eigenvalue inclusion sets derived in [37] and the new $Z$-eigenvalue localization sets for tensors are given. Additionally, the effects of orthonormal transformations for the proposed sets are investigated. As applications, some new bounds for the $Z$-spectral radius of weakly symmetric nonnegative tensors are established, which outperform some known results. Several numerical results illustrate these facts.

\section{TwO NEW $Z$-EIGENVALUE LOCALIZATION SETS FOR TENSORS}

In this section, we investigate $Z$-eigenvalue localization sets and present some new $Z$-eigenvalue localization sets for tensors, and the comparison results of the proposed sets with those in Lemmas 1.6, 1.8 and 1.9 are given. By making use of the technique applied in [22, Theorem 2.1], we establish the following theorem.

Theorem 2.1. Let $\mathcal{A}=\left(a_{i_{1} \ldots i_{m}}\right) \in \mathbb{C}^{[m, n]}$ and $n \geq 2$. Then all Z-eigenvalues of $\mathcal{A}$ are located in the following localization sets:

$$
\sigma(\mathcal{A}) \subseteq \mathcal{B}(\mathcal{A})=\left(\bigcup_{i \in N} \mathcal{B}_{i}^{1}(\mathcal{A})\right) \bigcup\left(\bigcup_{i, j \in N, i \neq j}\left(\mathcal{B}_{i, j}^{2}(\mathcal{A}) \bigcap \mathcal{K}_{i}(\mathcal{A})\right)\right)
$$

where

$$
\begin{gathered}
\mathcal{B}_{i}^{1}(\mathcal{A})=\left\{z \in \mathbb{C}:|z| \leq R_{i}(\mathcal{A})-P_{i}^{i}(\mathcal{A})\right\}, \quad \mathcal{K}_{i}(\mathcal{A})=\left\{z \in \mathbb{C}:|z| \leq R_{i}(\mathcal{A})\right\}, \\
B_{i, j}^{2}(\mathcal{A})=\left\{z \in \mathbb{C}:\left(|z|-\left[R_{i}(\mathcal{A})-P_{i}^{i}(\mathcal{A})\right]\right)\left(|z|-P_{j}^{i}(\mathcal{A})\right) \leq P_{i}^{i}(\mathcal{A})\left(R_{j}(\mathcal{A})-P_{j}^{i}(\mathcal{A})\right)\right\} .
\end{gathered}
$$

Proof. Let $\lambda$ be a $Z$-eigenvalue of $\mathcal{A}$ and the corresponding eigenvector be $x=$ $\left(x_{1}, x_{2}, \ldots, x_{n}\right)^{T} \in \mathbb{C}^{n} \backslash\{0\}$ with $\left|x_{t}\right| \geq\left|x_{s}\right| \geq \max \left\{\left|x_{k}\right|: k \in N, k \neq s, k \neq t\right\}$. Then it is evident that $\left|x_{t}\right|>0$ and

$$
\mathcal{A} x^{m-1}=\lambda x, \quad x^{T} x=1 .
$$

It follows from $(2.2)$ that

$$
\lambda x_{t}=\sum_{\substack{i_{2}, \ldots, i_{m} \in N, t \in\left\{i_{2}, \ldots, i_{m}\right\}}} a_{t i_{2} \ldots i_{m}} x_{i_{2}} \cdots x_{i_{m}}+\sum_{\substack{i_{2}, \ldots, i_{m} \in N \\ t \notin\left\{i_{2}, \ldots, i_{m}\right\}}} a_{t i_{2} \ldots i_{m}} x_{i_{2}} \cdots x_{i_{m}} .
$$


Since $\left|x_{t}\right|^{m-1} \leq\left|x_{t}\right| \leq 1,\left|x_{s}\right|^{m-1} \leq\left|x_{s}\right| \leq 1$, it holds that

$$
\begin{aligned}
|\lambda|\left|x_{t}\right| & \leq \sum_{\substack{i_{2}, \ldots, i_{m} \in N, t \in\left\{i_{2}, \ldots, i_{m}\right\}}}\left|a_{t i_{2} \ldots i_{m}}\right|\left|x_{i_{2}}\right| \cdots\left|x_{i_{m}}\right|+\sum_{\substack{i_{2}, \ldots, i_{m} \in N, t \notin\left\{i_{2}, \ldots, i_{m}\right\}}}\left|a_{t i_{2} \ldots i_{m}}\right|\left|x_{i_{2}}\right| \cdots\left|x_{i_{m}}\right| \\
& \leq \sum_{\substack{i_{2}, \ldots, i_{m} \in N, t \in\left\{i_{2}, \ldots, i_{m}\right\}}}\left|a_{t i_{2} \ldots i_{m}}\right|\left|x_{t}\right|^{m-1}+\sum_{\substack{i_{2}, \ldots, i_{m} \in N, t \notin\left\{i_{2}, \ldots, i_{m}\right\}}}\left|a_{t i_{2} \ldots i_{m}}\right|\left|x_{s}\right|^{m-1} \\
& \leq\left[R_{t}(\mathcal{A})-P_{t}^{t}(\mathcal{A})\right]\left|x_{t}\right|+P_{t}^{t}(\mathcal{A})\left|x_{s}\right|,
\end{aligned}
$$

which leads to

$$
|\lambda|-\left[R_{t}(\mathcal{A})-P_{t}^{t}(\mathcal{A})\right] \leq P_{t}^{t}(\mathcal{A}) \frac{\left|x_{s}\right|}{\left|x_{t}\right|} .
$$

If $\left|x_{s}\right|=0$, it follows from 2.3$)$ that $|\lambda|-\left[R_{t}(\mathcal{A})-P_{t}^{t}(\mathcal{A})\right] \leq 0$, that is, $|\lambda| \leq R_{t}(\mathcal{A})-$ $P_{t}^{t}(\mathcal{A})$. Evidently, $\lambda \in \mathcal{B}_{t}^{1}(\mathcal{A}) \subseteq \mathcal{B}(\mathcal{A})$. Otherwise, $\left|x_{s}\right|>0$. If $\lambda \notin \bigcup_{i \in N} \mathcal{B}_{i}^{1}(\mathcal{A})$, it is easy to see that for any $i \in N$,

$$
|\lambda|>R_{i}(\mathcal{A})-P_{i}^{i}(\mathcal{A})
$$

In particular, $|\lambda|>R_{t}(\mathcal{A})-P_{t}^{t}(\mathcal{A})$, i.e., $|\lambda|-\left[R_{t}(\mathcal{A})-P_{t}^{t}(\mathcal{A})\right]>0$. By $(3)$, it is not difficult to verify that $\lambda \in \mathcal{K}_{t}(\mathcal{A})$. Besides, it follows from 2.2 that

$$
\lambda x_{s}=\sum_{\substack{i_{2}, \ldots, i_{m} \in N, t \in\left\{i_{2}, \ldots, i_{m}\right\}}} a_{s i_{2} \ldots i_{m}} x_{i_{2}} \cdots x_{i_{m}}+\sum_{\substack{i_{2}, \ldots, i_{m} \in N, t \notin\left\{i_{2}, \ldots, i_{m}\right\}}} a_{s i_{2} \ldots i_{m}} x_{i_{2}} \cdots x_{i_{m}} .
$$

Taking absolute values in the above equation and using the triangle inequality yield

$$
\begin{aligned}
|\lambda|\left|x_{s}\right| & \leq \sum_{\substack{i_{2}, \ldots, i_{m} \in N, t \in\left\{i_{2}, \ldots, i_{m}\right\}}}\left|a_{s i_{2} \ldots i_{m}}\right|\left|x_{i_{2}}\right| \cdots\left|x_{i_{m}}\right|+\sum_{\substack{i_{2}, \ldots, i_{m} \in N, t \notin\left\{i_{2}, \ldots, i_{m}\right\}}}\left|a_{s i_{2} \ldots i_{m}}\right|\left|x_{i_{2}}\right| \cdots\left|x_{i_{m}}\right| \\
& \leq \sum_{\substack{i_{2}, \ldots, i_{m} \in N, t \in\left\{i_{2}, \ldots, i_{m}\right\}}}\left|a_{s i_{2} \ldots i_{m}}\right|\left|x_{t}\right|^{m-1}+\sum_{\substack{i_{2}, \ldots, i_{m} \in N, t \notin\left\{i_{2}, \ldots, i_{m}\right\}}}\left|a_{s i_{2} \ldots i_{m}}\right|\left|x_{s}\right|^{m-1} \\
& \leq\left[R_{s}(\mathcal{A})-P_{s}^{t}(\mathcal{A})\right]\left|x_{t}\right|+P_{s}^{t}(\mathcal{A})\left|x_{s}\right| .
\end{aligned}
$$

Recalling that $\left|x_{s}\right|>0$, then from inequality 2.4 we have

$$
|\lambda|-P_{s}^{t}(\mathcal{A}) \leq\left[R_{s}(\mathcal{A})-P_{s}^{t}(\mathcal{A})\right] \frac{\left|x_{t}\right|}{\left|x_{s}\right|} .
$$

Note that $|\lambda|-\left[R_{t}(\mathcal{A})-P_{t}^{t}(\mathcal{A})\right]>0$; multiplying 2.3 with 2.5 gives

$$
\left(|\lambda|-\left[R_{t}(\mathcal{A})-P_{t}^{t}(\mathcal{A})\right]\right)\left(|\lambda|-P_{s}^{t}(\mathcal{A})\right) \leq P_{t}^{t}(\mathcal{A})\left(R_{s}(\mathcal{A})-P_{s}^{t}(\mathcal{A})\right),
$$

which implies that $\lambda \in\left(\mathcal{B}_{t, s}^{2}(\mathcal{A}) \cap \mathcal{K}_{t}(\mathcal{A})\right) \subseteq \mathcal{B}(\mathcal{A})$. This completes our proof of Theorem 2.1.

The following theorem gives some comparisons between $\mathcal{B}(\mathcal{A})$ and the results in Lemmas $1.6,1.8$ and 1.9 To prove the relations, we need the following lemma. 
Lemma 2.2 ([17]). Let $a, b, c \geq 0$ and $d>0$.

(I) If $\frac{a}{b+c+d} \leq 1$, then

$$
\frac{a-(b+c)}{d} \leq \frac{a-b}{c+d} \leq \frac{a}{b+c+d}
$$

(II) If $\frac{a}{b+c+d} \geq 1$, then

$$
\frac{a-(b+c)}{d} \geq \frac{a-b}{c+d} \geq \frac{a}{b+c+d} .
$$

Theorem 2.3. Let $\mathcal{A}=\left(a_{i_{1} \ldots i_{m}}\right) \in \mathbb{C}^{[m, n]}$ and $n \geq 2$. Then

$$
\mathcal{B}(\mathcal{A}) \subseteq \mathcal{N}(\mathcal{A}) \subseteq \mathcal{K}(\mathcal{A}), \quad \mathcal{B}(\mathcal{A}) \subseteq \mathcal{M}(\mathcal{A}) \subseteq \mathcal{K}(\mathcal{A})
$$

Proof. We prove this theorem in view of the methods of [22, Theorem 2.3]. By Corollaries 3.2 and 3.3 in [37], it can be seen that $\mathcal{N}(\mathcal{A}) \subseteq \mathcal{K}(\mathcal{A})$ and $\mathcal{M}(\mathcal{A}) \subseteq$ $\mathcal{K}(\mathcal{A})$ hold true. Thus, we only need to show the relations $\mathcal{B}(\mathcal{A}) \subseteq \mathcal{N}(\mathcal{A})$ and $\mathcal{B}(\mathcal{A}) \subseteq \mathcal{M}(\mathcal{A})$. Now, we prove $\mathcal{B}(\mathcal{A}) \subseteq \mathcal{N}(\mathcal{A})$. Let $z \in \mathcal{B}(\mathcal{A})$. Then

$$
z \in \bigcup_{i \in N} \mathcal{B}_{i}^{1}(\mathcal{A}) \text { or } z \in \bigcup_{i, j \in N, i \neq j}\left(\mathcal{B}_{i, j}^{2}(\mathcal{A}) \bigcap \mathcal{K}_{i}(\mathcal{A})\right) .
$$

Without loss of generality, we first assume that $z \in \bigcup_{i \in N} \mathcal{B}_{i}^{1}(\mathcal{A})$. Under this condition, there exists one index $i_{0} \in N$ such that

$$
|z| \leq R_{i_{0}}(\mathcal{A})-P_{i_{0}}^{i_{0}}(\mathcal{A})
$$

i.e., $|z|-\left[R_{i_{0}}(\mathcal{A})-P_{i_{0}}^{i_{0}}(\mathcal{A})\right] \leq 0$. Hence, for any $j \neq i_{0}$, it follows that

$$
\left(|z|-\left[R_{i_{0}}(\mathcal{A})-P_{i_{0}}^{i_{0}}(\mathcal{A})\right]\right)|z| \leq P_{i_{0}}^{i_{0}}(\mathcal{A}) R_{j}(\mathcal{A}),
$$

which implies that $z \in \mathcal{N}_{i_{0}, j}(\mathcal{A}) \subseteq \mathcal{N}(\mathcal{A})$. If $z \notin \bigcup_{i \in N} \mathcal{B}_{i}^{1}(\mathcal{A})$, then for any $i \in N$, it holds that

$$
|z|>R_{i}(\mathcal{A})-P_{i}^{i}(\mathcal{A})
$$

and

$$
z \in \bigcup_{i, j \in N, i \neq j}\left(\mathcal{B}_{i, j}^{2}(\mathcal{A}) \bigcap \mathcal{K}_{i}(\mathcal{A})\right)
$$

It follows from 2.7 that there exist $p, q \in N$ and $p \neq q$ such that

$$
|z| \leq R_{p}(\mathcal{A})
$$

and

$$
\left(|z|-\left[R_{p}(\mathcal{A})-P_{p}^{p}(\mathcal{A})\right]\right)\left(|z|-P_{q}^{p}(\mathcal{A})\right) \leq P_{p}^{p}(\mathcal{A})\left(R_{q}(\mathcal{A})-P_{q}^{p}(\mathcal{A})\right) .
$$

If $P_{p}^{p}(\mathcal{A})\left(R_{q}(\mathcal{A})-P_{q}^{p}(\mathcal{A})\right)=0$, combining 2.6 and 2.9 results in

$$
|z|-P_{q}^{p}(\mathcal{A}) \leq 0 \leq R_{q}(\mathcal{A})-P_{q}^{p}(\mathcal{A})
$$

that is, $|z| \leq R_{q}(\mathcal{A})$, which is equivalent to

$$
|z|-\left[R_{q}(\mathcal{A})-P_{q}^{q}(\mathcal{A})\right] \leq P_{q}^{q}(\mathcal{A}) .
$$

By multiplying (2.8) with 2.10), we derive

$$
\left(|z|-\left[R_{q}(\mathcal{A})-P_{q}^{q}(\mathcal{A})\right]\right)|z| \leq P_{q}^{q}(\mathcal{A}) R_{p}(\mathcal{A}) .
$$


This means that $z \in \mathcal{N}_{q, p}(\mathcal{A}) \subseteq \mathcal{N}(\mathcal{A})$.

Afterwards, if $P_{p}^{p}(\mathcal{A})\left(R_{q}(\mathcal{A})-P_{q}^{p}(\mathcal{A})\right)>0$, dividing 2.9 by $P_{p}^{p}(\mathcal{A})\left(R_{q}(\mathcal{A})-\right.$ $\left.P_{q}^{p}(\mathcal{A})\right)$ yields

$$
\frac{\left(|z|-\left[R_{p}(\mathcal{A})-P_{p}^{p}(\mathcal{A})\right]\right)\left(|z|-P_{q}^{p}(\mathcal{A})\right)}{P_{p}^{p}(\mathcal{A})\left(R_{q}(\mathcal{A})-P_{q}^{p}(\mathcal{A})\right)} \leq 1 .
$$

Let $a=|z| \geq 0, b+c=P_{q}^{p}(\mathcal{A}) \geq 0$ with $b, c \geq 0$ and $d=R_{q}(\mathcal{A})-P_{q}^{p}(\mathcal{A})>0$. If $\frac{|z|-P_{q}^{p}(\mathcal{A})}{R_{q}(\mathcal{A})-P_{q}^{p}(\mathcal{A})} \geq 1$, then by 2.6 and (II) of Lemma 2.2 we have

$$
\frac{|z|-\left[R_{p}(\mathcal{A})-P_{p}^{p}(\mathcal{A})\right]}{P_{p}^{p}(\mathcal{A})} \frac{|z|}{R_{q}(\mathcal{A})} \leq \frac{|z|-\left[R_{p}(\mathcal{A})-P_{p}^{p}(\mathcal{A})\right]}{P_{p}^{p}(\mathcal{A})} \frac{|z|-P_{q}^{p}(\mathcal{A})}{R_{q}(\mathcal{A})-P_{q}^{p}(\mathcal{A})} \leq 1
$$

which is equivalent to

$$
\left(|z|-\left[R_{p}(\mathcal{A})-P_{p}^{p}(\mathcal{A})\right]\right)|z| \leq P_{p}^{p}(\mathcal{A}) R_{q}(\mathcal{A}) .
$$

This implies that $z \in \mathcal{N}_{p, q}(\mathcal{A}) \subseteq \mathcal{N}(\mathcal{A})$. Furthermore, if $\frac{|z|-P_{q}^{p}(\mathcal{A})}{R_{q}(\mathcal{A})-P_{q}^{p}(\mathcal{A})} \leq 1$, then 2.10 holds. Multiplying (2.8) with 2.10) leads to

$$
\left(|z|-\left[R_{q}(\mathcal{A})-P_{q}^{q}(\mathcal{A})\right]\right)|z| \leq P_{q}^{q}(\mathcal{A}) R_{p}(\mathcal{A}),
$$

which implies that $z \in \mathcal{N}_{q, p}(\mathcal{A}) \subseteq \mathcal{N}(\mathcal{A})$. Hence, $\mathcal{B}(\mathcal{A}) \subseteq \mathcal{N}(\mathcal{A})$.

In what follows, we show the relation $\mathcal{B}(\mathcal{A}) \subseteq \mathcal{M}(\mathcal{A})$. Similar to the above proof, we first suppose that $z \in \bigcup_{i \in N} \mathcal{B}_{i}^{1}(\mathcal{A})$, then there exists one index $i_{0} \in N$ such that $|z| \leq R_{i_{0}}(\mathcal{A})-P_{i_{0}}^{i_{0}}(\mathcal{A})$. For $j_{0} \in N$ and $j_{0} \neq i_{0}$, we have $\left|a_{i_{0} j_{0} \ldots j_{0}}\right| \leq P_{i_{0}}^{i_{0}}(\mathcal{A})$, from which it follows that

$$
|z| \leq R_{i_{0}}(\mathcal{A})-P_{i_{0}}^{i_{0}}(\mathcal{A}) \leq R_{i_{0}}(\mathcal{A})-\left|a_{i_{0} j_{0} \ldots j_{0}}\right| .
$$

For $j_{0} \in N$ and $j_{0} \neq i_{0}$, it holds that

$$
|z|<P_{j_{0}}^{i_{0}}(\mathcal{A}) \quad \text { or } \quad|z| \geq P_{j_{0}}^{i_{0}}(\mathcal{A}) .
$$

If $|z| \geq P_{j_{0}}^{i_{0}}(\mathcal{A})$, then we have

$$
\left[|z|-\left(R_{i_{0}}(\mathcal{A})-\left|a_{i_{0} j_{0} \ldots j_{0}}\right|\right)\right]\left(|z|-P_{j_{0}}^{i_{0}}(\mathcal{A})\right) \leq 0 \leq\left|a_{i_{0} j_{0} \ldots j_{0}}\right|\left(R_{j_{0}}(\mathcal{A})-P_{j_{0}}^{i_{0}}(\mathcal{A})\right),
$$

which implies that $z \in \mathcal{M}_{i_{0}, j_{0}}(\mathcal{A}) \subseteq \mathcal{M}(\mathcal{A})$. Otherwise, $|z|<P_{j_{0}}^{i_{0}}(\mathcal{A})$. Under this condition, if $|z|=R_{i_{0}}(\mathcal{A})-\left|a_{i_{0} j_{0} \ldots j_{0}}\right|$, then it is evident that $z \in \mathcal{M}_{i_{0}, j_{0}}(\mathcal{A}) \subseteq$ $\mathcal{M}(\mathcal{A})$. If $|z|<R_{i_{0}}(\mathcal{A})-\left|a_{i_{0} j_{0} \ldots j_{0}}\right|$, then $z \in \mathcal{H}_{i_{0}, j_{0}}(\mathcal{A}) \subseteq \mathcal{M}(\mathcal{A})$.

If $z \notin \bigcup_{i \in N} \mathcal{B}_{i}^{1}(\mathcal{A})$, then 2.6$)-(2.9)$ hold true. If $P_{p}^{p}(\mathcal{A})\left(R_{q}(\mathcal{A})-P_{q}^{p}(\mathcal{A})\right)=0$, then $P_{p}^{p}(\mathcal{A})=0$ or $R_{q}(\mathcal{A})=P_{q}^{p}(\mathcal{A})$. When $P_{p}^{p}(\mathcal{A})=0$, then $\left|a_{p q \ldots q}\right|=0$ and therefore

$$
\begin{aligned}
& \left(|z|-\left[R_{p}(\mathcal{A})-\left|a_{p q \ldots q}\right|\right]\right)\left(|z|-P_{q}^{p}(\mathcal{A})\right) \\
& \quad=\left(|z|-\left[R_{p}(\mathcal{A})-P_{p}^{p}(\mathcal{A})\right]\right)\left(|z|-P_{q}^{p}(\mathcal{A})\right) \\
& \quad \leq P_{p}^{p}(\mathcal{A})\left(R_{q}(\mathcal{A})-P_{q}^{p}(\mathcal{A})\right)=\left|a_{p q \ldots q}\right|\left(R_{q}(\mathcal{A})-P_{q}^{p}(\mathcal{A})\right),
\end{aligned}
$$

which implies that $z \in \mathcal{M}_{p, q}(\mathcal{A}) \subseteq \mathcal{M}(\mathcal{A})$.

If $R_{q}(\mathcal{A})=P_{q}^{p}(\mathcal{A})$, then it follows from $|z|>R_{p}(\mathcal{A})-P_{p}^{p}(\mathcal{A})$ and inequality $(2.9)$ that $|z| \leq P_{q}^{p}(\mathcal{A})$. If $|z|=P_{q}^{p}(\mathcal{A})$, then it is obvious that $z \in \mathcal{M}_{p, q}(\mathcal{A}) \subseteq \mathcal{M}(\mathcal{A})$. 
Otherwise, $|z|<P_{q}^{p}(\mathcal{A})$. Under this case, if $|z|-\left[R_{p}(\mathcal{A})-P_{p}^{p}(\mathcal{A})\right]<0$, then $z \in \mathcal{H}_{p, q}(\mathcal{A}) \subseteq \mathcal{M}(\mathcal{A})$. If $|z|-\left[R_{p}(\mathcal{A})-P_{p}^{p}(\mathcal{A})\right] \geq 0$, then

$$
\left[|z|-\left(R_{p}(\mathcal{A})-\left|a_{p q \ldots q}\right|\right)\right]\left(|z|-P_{q}^{p}(\mathcal{A})\right) \leq 0=\left|a_{p q \ldots q}\right|\left(R_{q}(\mathcal{A})-P_{q}^{p}(\mathcal{A})\right),
$$

which means that $z \in \mathcal{M}_{p, q}(\mathcal{A}) \subseteq \mathcal{M}(\mathcal{A})$.

If $P_{p}^{p}(\mathcal{A})\left(R_{q}(\mathcal{A})-P_{q}^{p}(\mathcal{A})\right)>0$, then

$$
\frac{|z|-\left[R_{p}(\mathcal{A})-P_{p}^{p}(\mathcal{A})\right]}{P_{p}^{p}(\mathcal{A})} \frac{|z|-P_{q}^{p}(\mathcal{A})}{R_{q}(\mathcal{A})-P_{q}^{p}(\mathcal{A})} \leq 1 .
$$

Since $|z| \leq R_{p}(\mathcal{A})$, we have $\frac{|z|-\left[R_{p}(\mathcal{A})-P_{p}^{p}(\mathcal{A})\right]}{P_{p}^{p}(\mathcal{A})} \leq 1$. If $|z|<P_{q}^{p}(\mathcal{A})$ and $|z|<R_{p}(\mathcal{A})-$ $\left|a_{p q \ldots q}\right|$, then $z \in \mathcal{H}_{p, q}(\mathcal{A}) \subseteq \mathcal{M}(\mathcal{A})$. If $|z|<P_{q}^{p}(\mathcal{A})$ and $|z| \geq R_{p}(\mathcal{A})-\left|a_{p q \ldots q}\right|$, then $z \in \mathcal{M}_{p, q}(\mathcal{A}) \subseteq \mathcal{M}(\mathcal{A})$. If $|z| \geq P_{q}^{p}(\mathcal{A})$ and $\left|a_{p q \ldots q}\right|>0$, then let $|z|=a \geq 0$, $b=R_{p}(A)-P_{p}^{p}(A) \geq 0, c=P_{p}^{p}(A)-\left|a_{p q \ldots q}\right| \geq 0$, and $d=\left|a_{p q \ldots q}\right|$. Thus, from inequality (2.11), we have that

$$
\begin{array}{ll}
\frac{|z|-\left[R_{p}(\mathcal{A})-\left|a_{p q \ldots q}\right|\right]}{\left|a_{p q \ldots q}\right|} \frac{|z|-P_{q}^{p}(\mathcal{A})}{R_{q}(\mathcal{A})-P_{q}^{p}(\mathcal{A})} \\
\quad \leq \frac{|z|-\left[R_{p}(\mathcal{A})-P_{p}^{p}(\mathcal{A})\right]}{P_{p}^{p}(\mathcal{A})} \frac{|z|-P_{q}^{p}(\mathcal{A})}{R_{q}(\mathcal{A})-P_{q}^{p}(\mathcal{A})} \leq 1,
\end{array}
$$

by virtue of Lemma 2.2 From inequality 2.12 we derive

$$
\left(|z|-\left[R_{p}(\mathcal{A})-\left|a_{p q \ldots q}\right|\right]\right)\left(|z|-P_{q}^{p}(\mathcal{A})\right) \leq\left|a_{p q \ldots q}\right|\left(R_{q}(\mathcal{A})-P_{q}^{p}(\mathcal{A})\right),
$$

from which it follows that $z \in \mathcal{M}_{p, q}(\mathcal{A}) \subseteq \mathcal{M}(\mathcal{A})$. If $|z| \geq P_{q}^{p}(\mathcal{A})$ and $\left|a_{p q \ldots q}\right|=0$, then $|z| \leq R_{p}(\mathcal{A})=R_{p}(\mathcal{A})-\left|a_{p q \ldots q}\right|$, which leads to

$$
\begin{aligned}
\left(|z|-\left[R_{p}(\mathcal{A})-\right.\right. & \left.\left.\left|a_{p q \ldots q}\right|\right]\right)\left(|z|-P_{q}^{p}(\mathcal{A})\right) \\
& =\left(|z|-R_{p}(\mathcal{A})\right)\left(|z|-P_{q}^{p}(\mathcal{A})\right) \leq 0=\left|a_{p q \ldots q}\right|\left(R_{q}(\mathcal{A})-P_{q}^{p}(\mathcal{A})\right) .
\end{aligned}
$$

This implies that $z \in \mathcal{M}_{p, q}(\mathcal{A}) \subseteq \mathcal{M}(\mathcal{A})$. Therefore, $\mathcal{B}(\mathcal{A}) \subseteq \mathcal{M}(\mathcal{A})$. The conclusions follow immediately from what we have proved.

Thus, the $Z$-eigenvalue localization set of Theorem 2.1 is more precise than those in Lemmas $1.6,1.8$ and 1.9 . Now we give an example to verify the fact.

Example 2.4 $([37])$. Consider the tensor $\mathcal{A}=\left(a_{i j k}\right) \in \mathbb{R}^{[3,3]}$ defined by

$$
a_{i j k}=\left\{\begin{array}{l}
a_{111}=1 ; a_{112}=-1 ; a_{131}=1 ; a_{133}=1 ; \\
a_{211}=-1 ; a_{222}=2 ; a_{232}=1 ; \\
a_{311}=1 ; a_{323}=1 ; a_{333}=3 \\
a_{i j k}=0, \text { otherwise. }
\end{array}\right.
$$

By some calculation, the comparative results are given in Table 1 As observed in this table, $\mathcal{B}(\mathcal{A})$ is the sharpest among the aforementioned localization sets.

In the following theorem, motivated by [1, Theorem 3.3], we develop another $Z$-eigenvalue localization set for tensors. 
TABLE 1 . Some $Z$-eigenvalue localization sets for $\mathcal{A}$.

\begin{tabular}{ll}
\hline Theorem 3.1 of [37] & $\mathcal{K}(\mathcal{A})=\{z \in \mathbb{C}:|z| \leq 5\}$ \\
\hline Theorem 3.3 of [37] & $\mathcal{M}(\mathcal{A})=\{z \in \mathbb{C}:|z| \leq 5\}$ \\
\hline Theorem 3.4 of [37] & $\mathcal{N}(\mathcal{A})=\{z \in \mathbb{C}:|z| \leq 2+2 \sqrt{2}\}$ \\
\hline Theorem 2.1 & $\mathcal{B}(\mathcal{A})=\{z \in \mathbb{C}:|z| \leq 4.7913\}$ \\
\hline
\end{tabular}

Theorem 2.5. Let $\mathcal{A}=\left(a_{i_{1} \ldots i_{m}}\right) \in \mathbb{C}^{[m, n]}, m, n \geq 2$ and $R_{i}(\mathcal{A})>0$ for $i \in N$. Then all $Z$-eigenvalues of $\mathcal{A}$ are located in the following localization sets:

$$
\sigma(\mathcal{A}) \subseteq \mathcal{F}(\mathcal{A})=\bigcup_{a_{i_{1} i_{2} \ldots i_{m}} \neq 0}\left\{z \in \mathbb{C}:|z| \leq\left(\prod_{l=1}^{m} R_{i_{l}}(\mathcal{A})\right)^{\frac{1}{m}}\right\}
$$

Proof. Let $\lambda$ be a $Z$-eigenvalue of $\mathcal{A}$ with the corresponding eigenvector $x$, i.e., $\mathcal{A} x^{m-1}=\lambda x$. Let $\left|x_{\alpha}\right|=\max \left\{\left|x_{i_{1}}\right|\left|x_{i_{2}}\right| \cdots\left|x_{i_{m}}\right|: a_{i_{1} i_{2} \ldots i_{m}} \neq 0, i_{1}, \ldots, i_{m} \in N\right\}$. Then for all $i \in N$, it holds that

$$
\begin{aligned}
\left|\lambda x_{i}^{2}\right| & =\left|\sum_{i_{2}, \ldots, i_{m}=1}^{n} a_{i i_{2} \ldots i_{m}} x_{i} x_{i_{2}} \cdots x_{i_{m}}\right| \\
& \leq \sum_{i_{2}, \ldots, i_{m}=1}^{n}\left|a_{i i_{2} \ldots i_{m}}\right|\left|x_{i}\right|\left|x_{i_{2}}\right| \cdots\left|x_{i_{m}}\right| \\
& =\sum_{a_{i i_{2} \ldots i_{m}} \neq 0}\left|a_{i i_{2} \ldots i_{m}}\right|\left|x_{i}\right|\left|x_{i_{2}}\right| \cdots\left|x_{i_{m}}\right| \leq R_{i}(\mathcal{A})\left|x_{\alpha}\right| .
\end{aligned}
$$

Now, we consider two cases as follows.

(i) $\left|x_{\alpha}\right|=0$. Since $x \neq 0$, suppose that $x_{p} \neq 0, p \in N$. Then from 2.13), we have $\left|\lambda x_{p}^{2}\right|=0$, i.e., $\lambda=0$. Clearly, $\lambda \in \mathcal{F}(\mathcal{A})$.

(ii) $\left|x_{\alpha}\right|>0$. Suppose that $\left|x_{\alpha}\right|=\left|x_{j_{1}}\right|\left|x_{j_{2}}\right| \cdots\left|x_{j_{m}}\right|$. Then from (2.13), we obtain the inequalities

$$
\begin{gathered}
|\lambda|\left|x_{j_{1}}\right|^{2} \leq R_{j_{1}}(\mathcal{A})\left|x_{\alpha}\right|, \\
\vdots \\
|\lambda|\left|x_{j_{m}}\right|^{2} \leq R_{j_{m}}(\mathcal{A})\left|x_{\alpha}\right| .
\end{gathered}
$$

Then

which yields that

$$
\prod_{l=1}^{m}|\lambda|\left|x_{j_{l}}\right|^{2} \leq\left|x_{\alpha}\right|^{m} \prod_{l=1}^{m} R_{j_{l}}(\mathcal{A})
$$

$$
\left|x_{\alpha}\right|^{2} \prod_{l=1}^{m}|\lambda| \leq\left|x_{\alpha}\right|^{m} \prod_{l=1}^{m} R_{j_{l}}(\mathcal{A})
$$


and therefore

$$
\prod_{l=1}^{m}|\lambda| \leq\left|x_{\alpha}\right|^{m-2} \prod_{l=1}^{m} R_{j_{l}}(\mathcal{A}) \leq \prod_{l=1}^{m} R_{j_{l}}(\mathcal{A})
$$

by $m \geq 2$ and $\left|x_{\alpha}\right| \leq 1$. Hence, we obtain

$$
\sigma(\mathcal{A}) \subseteq \mathcal{F}(\mathcal{A})=\bigcup_{a_{i_{1} i_{2} \ldots i_{m}} \neq 0}\left\{z \in \mathbb{C}:|z| \leq\left(\prod_{l=1}^{m} R_{i_{l}}(\mathcal{A})\right)^{\frac{1}{m}}\right\} .
$$

The proof is completed.

In the following theorem we show that $\mathcal{F}(\mathcal{A}) \subseteq \mathcal{K}(\mathcal{A})$.

Theorem 2.6. Let $\mathcal{A}=\left(a_{i_{1} \ldots i_{m}}\right) \in \mathbb{C}^{[m, n]}, m, n \geq 2$ and $R_{i}(\mathcal{A})>0$ for $i \in N$. Then $\mathcal{F}(\mathcal{A}) \subseteq \mathcal{K}(\mathcal{A})$.

Proof. For any $z \in \mathcal{F}(\mathcal{A})$, if $z \notin \mathcal{K}(\mathcal{A})$, then $|z|>R_{i}(\mathcal{A})$ for all $i \in N$. In this case, for all $a_{i_{1} i_{2} \ldots i_{m}} \neq 0, i_{j} \in N$ and $j=1,2, \ldots, m$, it follows that

$$
|z|>\left(\prod_{l=1}^{m} R_{j_{l}}(\mathcal{A})\right)^{\frac{1}{m}}
$$

which contradicts the fact that $z \in \mathcal{F}(\mathcal{A})$.

Next, enlightened by [19], we investigate the relationships of $\mathcal{F}(\mathcal{A}), \mathcal{K}(\mathcal{A})$, and $\mathcal{N}(\mathcal{A})$.

Theorem 2.7. Let $\mathcal{A}=\left(a_{i_{1} \ldots i_{m}}\right) \in \mathbb{C}^{[m, n]}$ with $m, n \geq 2$. If $a_{i \ldots i} \neq 0$ for each $i \in N$, then $\mathcal{N}(\mathcal{A}) \subseteq \mathcal{F}(\mathcal{A})=\mathcal{K}(\mathcal{A})$.

Proof. If $a_{i \ldots i} \neq 0$ for each $i \in N$, then

$$
\begin{aligned}
\bigcup_{i \in N}\left\{z \in \mathbb{C}:|z| \leq\left(R_{i}(\mathcal{A})^{m}\right)^{\frac{1}{m}}\right. & \left.=R_{i}(\mathcal{A})\right\}=\mathcal{K}(\mathcal{A}) \\
\subseteq \mathcal{F}(\mathcal{A}) & =\bigcup_{a_{i_{1} i_{2} \ldots i_{m}} \neq 0}\left\{z \in \mathbb{C}:|z| \leq\left(\prod_{l=1}^{m} R_{i_{l}}(\mathcal{A})\right)^{\frac{1}{m}}\right\},
\end{aligned}
$$

which together with $\mathcal{F}(\mathcal{A}) \subseteq \mathcal{K}(\mathcal{A})$ results in $\mathcal{F}(\mathcal{A})=\mathcal{K}(\mathcal{A})$. According to Theorem 2.2, we obtain $\mathcal{N}(\mathcal{A}) \subseteq \mathcal{F}(\mathcal{A})=\mathcal{K}(\mathcal{A})$.

Now, we utilize the following example to confirm the result in Theorem 2.5.

Example 2.8. Consider the tensor $\mathcal{A}=\left(a_{i j k}\right) \in \mathbb{R}^{[3,2]}$ defined by

$$
a_{i j k}=\left\{\begin{array}{l}
a_{111}=2 ; a_{222}=1 ; \\
a_{112}=a_{122}=a_{211}=-\frac{4}{3} ; \\
a_{i j k}=0, \text { otherwise. }
\end{array}\right.
$$


TABLE 2. Some $Z$-eigenvalue localization sets for $\mathcal{A}$.

\begin{tabular}{cc}
\hline $\mathcal{K}(\mathcal{A})$ & $\{z \in \mathbb{C}:|z| \leq 4.6667\}$ \\
\hline $\mathcal{N}(\mathcal{A})$ & $\{z \in \mathbb{C}:|z| \leq 4.0934\}$ \\
\hline $\mathcal{B}(\mathcal{A})$ & $\{z \in \mathbb{C}:|z| \leq 3.9384\}$ \\
\hline $\mathcal{F}(\mathcal{A})$ & $\{z \in \mathbb{C}:|z| \leq 4.6667\}$ \\
\hline
\end{tabular}

Obviously, $a_{111}, a_{222} \neq 0$. We present the comparative results in Table 2 As seen in this table, the relationship $\mathcal{N}(\mathcal{A}) \subseteq \mathcal{F}(\mathcal{A})=\mathcal{K}(\mathcal{A})$ holds; what is more, we also get $\mathcal{B}(\mathcal{A}) \subseteq \mathcal{F}(\mathcal{A})$.

Theorem 2.9. Let $\mathcal{A}=\left(a_{i_{1} \ldots i_{m}}\right) \in \mathbb{C}^{[m, n]}, m, n \geq 2$ and $R_{i}(\mathcal{A}) \neq 0$. If $R_{i}(\mathcal{A})=$ $P_{i}^{i}(\mathcal{A})$ for each $i \in N$, then $\mathcal{F}(\mathcal{A}) \subseteq \mathcal{N}(\mathcal{A}) \subseteq \mathcal{K}(\mathcal{A})$.

Proof. It follows from $R_{i}(\mathcal{A})=P_{i}^{i}(\mathcal{A})$ for each $i \in N$ that $a_{i \ldots i}=0$ for all $i \in N$ and

$$
\begin{aligned}
\mathcal{N}(\mathcal{A}) & =\bigcup_{i, j \in N, i \neq j}\left\{z \in \mathbb{C}:\left(|z|-\left[R_{i}(\mathcal{A})-P_{i}^{i}(\mathcal{A})\right]\right)|z| \leq P_{i}^{i}(\mathcal{A}) R_{j}(\mathcal{A})\right\} \\
& =\bigcup_{i, j \in N, i \neq j}\left\{z \in \mathbb{C}:|z|^{2} \leq R_{i}(\mathcal{A}) R_{j}(\mathcal{A})\right\} .
\end{aligned}
$$

Let $\lambda$ be any eigenvalue of the tensor $\mathcal{A}$. By Theorem 2.5 and $R_{i}(\mathcal{A}) \neq 0$ for all $i \in N$, there exists $a_{h_{1} \ldots h_{m}} \neq 0$ such that

$$
|\lambda| \leq\left(\prod_{l=1}^{m} R_{h_{l}}(\mathcal{A})\right)^{\frac{1}{m}}
$$

On squaring the above inequality, we have $|\lambda|^{2 m} \leq \prod_{l=1}^{m}\left[R_{h_{l}}(\mathcal{A})\right]^{2}$. Since $R_{i}(\mathcal{A}) \neq 0$ for all $i \in N$, the inequality $|\lambda|^{2 m} \leq \prod_{l=1}^{m}\left[R_{h_{l}}(\mathcal{A})\right]^{2}$ can be rewritten as

$$
\left(\frac{|\lambda|^{2}}{R_{h_{1}}(\mathcal{A}) R_{h_{2}}(\mathcal{A})}\right) \cdots\left(\frac{|\lambda|^{2}}{R_{h_{m}}(\mathcal{A}) R_{h_{1}}(\mathcal{A})}\right) \leq 1 .
$$

It follows from this inequality that at least one of the factors in its left-hand side is at most 1 . Without loss of generality, we assume that there exists $p$ with $1 \leq p \leq n$ such that

$$
|\lambda|^{2} \leq R_{h_{p}}(\mathcal{A}) R_{h_{p+1}}(\mathcal{A}),
$$

where if $p=m$, then $h_{p+1}=h_{1}$. This inequality implies that

$$
\lambda \in\left\{z \in \mathbb{C}:|z|^{2} \leq R_{h_{p}}(\mathcal{A}) R_{h_{p+1}}(\mathcal{A})\right\} \subseteq \mathcal{N}(\mathcal{A}) .
$$

As a result, we derive $\mathcal{F}(\mathcal{A}) \subseteq \mathcal{N}(\mathcal{A}) \subseteq \mathcal{K}(\mathcal{A})$ by virtue of Theorem 2.3 The result follows. 
The following example illustrates the relationship $\mathcal{F}(\mathcal{A}) \subseteq \mathcal{N}(\mathcal{A}) \subseteq \mathcal{K}(\mathcal{A})$ in Theorem 2.9 .

Example 2.10. Let $\mathcal{A}=\left(a_{i_{1} i_{2} i_{3}}\right) \in \mathbb{R}^{[3,3]}$, where $a_{123}=1, a_{132}=a_{211}=2$, $a_{213}=a_{321}=1.5, a_{312}=2.5$, and the other entries are zero.

By computations, it is not difficult to verify that $R_{i}(\mathcal{A})=P_{i}^{i}(\mathcal{A}) \neq 0(i=1,2,3)$. We compare the $Z$-eigenvalue localization sets $\mathcal{B}(\mathcal{A})$ and $\mathcal{F}(\mathcal{A})$ with $\mathcal{N}(\mathcal{A})$ and $\mathcal{K}(\mathcal{A})$ in Table 3 . From the observations in this table, it can be clearly seen that $\mathcal{F}(\mathcal{A}) \subseteq \mathcal{N}(\mathcal{A}) \subseteq \mathcal{K}(\mathcal{A})$ and $\mathcal{F}(\mathcal{A}) \subseteq \mathcal{B}(\mathcal{A})$.

TABLE 3. Some $Z$-eigenvalue localization sets for $\mathcal{A}$.

\begin{tabular}{ll}
\hline $\mathcal{K}(\mathcal{A})$ & $\{z \in \mathbb{C}:|z| \leq 4\}$ \\
\hline $\mathcal{N}(\mathcal{A})$ & $\{z \in \mathbb{C}:|z| \leq 3.7417\}$ \\
\hline $\mathcal{B}(\mathcal{A})$ & $\{z \in \mathbb{C}:|z| \leq 3.7417\}$ \\
\hline $\mathcal{F}(\mathcal{A})$ & $\{z \in \mathbb{C}:|z| \leq 3.4760\}$ \\
\hline
\end{tabular}

Remark 2.11. From Tables 2 and 3 , we observe that the set $\mathcal{B}(\mathcal{A})$ or the set $\mathcal{F}(\mathcal{A})$ can work better than the other one. Here, an interesting problem arises: What is the relationship between the set $\mathcal{B}(\mathcal{A})$ and the set $\mathcal{F}(\mathcal{A})$ ? In the future, we will research this problem.

\section{Orthogonal transformation for the $Z$-Eigenvalue LOCALiZATion SETS OF TENSORS}

It is noteworthy that the $Z$-eigenvalues of the tensors are invariant under orthonormal transformations 32 and have applications in physics and mechanics. Thus it is meaningful to analyze whether $Z$-eigenvalue localization sets for tensors obtained in Theorems 2.1 and 2.5 are invariant under orthonormal transformations, or what are the effects of orthonormal transformations. In physics and mechanics, it is most important that the results are independent from coordinate system choice. Now we introduce the definition of the orthogonal transformation for tensors.

Definition 3.1 ([32]). For any given $n \times n$ real orthogonal matrix $P=\left(p_{i j}\right)$ and tensor $\mathcal{A}=\left(a_{i_{1} \ldots i_{m}}\right) \in \mathbb{C}^{[m, n]}$, the orthogonal transformation $P^{m}(\mathcal{A})$ is defined as

$$
P^{m}(\mathcal{A})=\left(\sum_{i_{1}, i_{2}, \ldots, i_{m}=1}^{n} a_{i_{1} \ldots i_{m}} p_{j_{1} i_{1}} \cdots p_{j_{m} i_{m}}\right) \in \mathbb{C}^{[m, n]} .
$$

For any given two tensors $\mathcal{A}=\left(a_{i_{1} \ldots i_{m}}\right), \mathcal{D}=\left(d_{j_{1} \ldots j_{m}}\right) \in \mathbb{C}^{[m, n]}$, we say that $\mathcal{A}$ is orthogonally similar to $\mathcal{D}$ if there exists some orthogonal matrix $P$ such that $\mathcal{D}=P^{m}(\mathcal{A})$.

The following lemma shows that the $Z$-eigenvalues of the tensors are invariant under orthonormal transformations. 
Lemma $3.2\left([32)\right.$. Let $\mathcal{A}=\left(a_{i_{1} \ldots i_{m}}\right), \mathcal{D}=\left(d_{j_{1} \ldots j_{m}}\right) \in \mathbb{C}^{[m, n]}$ be orthogonally similar with $\mathcal{D}=P^{m}(\mathcal{A})$, where $P=\left(p_{i j}\right)$ is an $n \times n$ orthogonal matrix. Then $\mathcal{A}$ and $\mathcal{D}$ have the same $Z$-eigenvalues. In particular, if $\lambda$ is a $Z$-eigenvalue of $\mathcal{A}$ with a $Z$-eigenvector $\boldsymbol{x}$, then $\lambda$ is also a $Z$-eigenvalue of $\mathcal{D}$ with a $Z$-eigenvector $\boldsymbol{y}=P \boldsymbol{x}$.

Next, we discuss whether $Z$-eigenvalue localization sets for tensors obtained in Theorems 2.1 and 2.5 are invariant under orthonormal transformations, or what are the effects of orthonormal transformations.

For a given orthogonal matrix $P=\left(p_{i j}\right) \in \mathbb{R}^{n \times n}, \mathcal{D}=P^{m}(\mathcal{A})$. Based on Lemma 3.2 and applying the same technique used in Theorem 2.1, we can derive

$$
\sigma(\mathcal{A})=\sigma(\mathcal{D}) \subseteq \overline{\mathcal{B}}(\mathcal{D})=\left(\bigcup_{i \in N} \overline{\mathcal{B}}_{i}^{1}(\mathcal{D})\right) \bigcup\left(\bigcup_{i, j \in N, i \neq j}\left(\overline{\mathcal{B}}_{i, j}^{2}(\mathcal{D}) \bigcap \overline{\mathcal{K}}_{i}(\mathcal{D})\right)\right)
$$

where

$$
\begin{gathered}
\overline{\mathcal{B}}_{i}^{1}(\mathcal{D})=\left\{z \in \mathbb{C}:|z| \leq R_{i}(\mathcal{D})-P_{i}^{i}(\mathcal{D})\right\}, \quad \overline{\mathcal{K}}_{i}(\mathcal{D})=\left\{z \in \mathbb{C}:|z| \leq R_{i}(\mathcal{D})\right\} \\
\bar{B}_{i, j}^{2}(\mathcal{D})=\left\{z \in \mathbb{C}:\left(|z|-\left[R_{i}(\mathcal{D})-P_{i}^{i}(\mathcal{D})\right]\right)\left(|z|-P_{j}^{i}(\mathcal{D})\right) \leq P_{i}^{i}(\mathcal{D})\left(R_{j}(\mathcal{D})-P_{j}^{i}(\mathcal{D})\right)\right\} .
\end{gathered}
$$

Similarly, we can establish the following $Z$-eigenvalue localization set by virtue of Theorem 2.5.

$$
\sigma(\mathcal{A})=\sigma(\mathcal{D}) \subseteq \overline{\mathcal{F}}(\mathcal{D})=\bigcup_{d_{i_{1} i_{2} \ldots i_{m}} \neq 0}\left\{z \in \mathbb{C}:|z| \leq\left(\prod_{l=1}^{m} R_{i_{l}}(\mathcal{D})\right)^{\frac{1}{m}}\right\} .
$$

Now we illustrate that the $Z$-eigenvalue localization sets for tensors obtained in Theorems 2.1 and 2.5 are variant under orthonormal transformations.

For Example 2.4, we choose the orthogonal matrix generated by the function orth of Matlab

$$
P=\left(\begin{array}{ccc}
-0.6612 & -0.4121 & -0.6269 \\
-0.6742 & -0.0400 & 0.7375 \\
-0.3290 & 0.9103 & -0.2513
\end{array}\right)
$$

By 3.1 , we obtain $\overline{\mathcal{B}}(\mathcal{D})=\{z \in \mathbb{C}:|z| \leq 7.1733\}$, while $\mathcal{B}(\mathcal{A})=\{z \in \mathbb{C}$ : $|z| \leq 4.7913\}$ in terms of Theorem 2.1. This means that $\mathcal{B}(\mathcal{A})$ (no orthonormal transformations) is better than $\overline{\mathcal{B}}(\mathcal{D})$.

For Example 2.10 we also consider the orthogonal matrix $(3.3)$; then we have the $Z$-eigenvalue localization set $\overline{\mathcal{F}}(\mathcal{D})=\{z \in \mathbb{C}:|z| \leq 6.1935\}$ by Theorem 2.5 under this orthonormal transformation, which means that $\mathcal{F}(\mathcal{A})$ also becomes worse under this orthonormal transformation.

The following example indicates that under orthonormal transformations, the $Z$-eigenvalue localization sets obtained in this paper may become sharper. 
Example 3.3. Consider the tensor $\mathcal{A}=\left(a_{i_{1} i_{2} i_{3}}\right) \in \mathbb{R}^{[3,3]}$ generated by Matlab randomly, where

$$
\begin{aligned}
& \mathcal{A}(:,:, 1)=\left(\begin{array}{lll}
0.6948 & 0.3171 & 0.9502 \\
0.4898 & 0.4456 & 0.6463 \\
0.1190 & 0.4984 & 0.9597
\end{array}\right), \\
& \mathcal{A}(:,:, 2)=\left(\begin{array}{lll}
0.0344 & 0.4387 & 0.3816 \\
0.7094 & 0.7547 & 0.2760 \\
0.3404 & 0.5853 & 0.2238
\end{array}\right), \\
& \mathcal{A}(:,:, 3)=\left(\begin{array}{lll}
0.7655 & 0.7952 & 0.1869 \\
0.6797 & 0.6551 & 0.1626 \\
0.7513 & 0.2551 & 0.5060
\end{array}\right) .
\end{aligned}
$$

We report in Table 4 the $Z$-eigenvalue localization sets for $\mathcal{A}$ by Theorem 2.1. (3.1), Theorem 2.5, and (3.2). By comparing the results in this table, we conclude that under orthonormal transformations with the orthogonal matrix (3.3), the sets derived in Theorems 2.1 and 2.5 become better, and it implies that orthonormal transformations may make the $Z$-eigenvalue localization sets sharper.

TABlE 4 . Some $Z$-eigenvalue localization sets for $\mathcal{A}$.

\begin{tabular}{ll}
\hline $\mathcal{B}(\mathcal{A})$ & $\{z \in \mathbb{C}:|z| \leq 4.7162\}$ \\
\hline$\overline{\mathcal{B}}(\mathcal{D})$ & $\{z \in \mathbb{C}:|z| \leq 3.6766\}$ \\
\hline $\mathcal{F}(\mathcal{A})$ & $\{z \in \mathbb{C}:|z| \leq 4.8192\}$ \\
\hline$\overline{\mathcal{F}}(\mathcal{D})$ & $\{z \in \mathbb{C}:|z| \leq 4.6232\}$ \\
\hline
\end{tabular}

In summary, the $Z$-eigenvalue localization sets for tensors obtained in this paper are variant under orthonormal transformations. Under proper orthonormal transformations, they may be better. An interesting problem is: How to choose the orthogonal matrix $P$ such that the $Z$-eigenvalue localization sets are best under orthonormal transformations. It is a difficult task for us at present, and we will investigate this problem in our further work.

\section{SOME NEW UPPER BOUNDS FOR THE $Z$-SPECTRAL RADIUS OF WEAKLY SYMMETRIC NONNEGATIVE TENSORS}

On the basis of the results in Section 2 we establish two new upper bounds for the $Z$-spectral radius of weakly symmetric tensors in this section, and compare those with some known bounds derived in [36, 11, 24, 37]. To this end, we start with a lemma which will be useful in the following proofs.

Lemma 4.1 ([37]). Assume $\mathcal{A}$ is a weakly symmetric nonnegative tensor. Then $\rho(\mathcal{A})=\lambda^{*}$, where $\lambda^{*}$ denotes the largest $Z$-eigenvalue. 
Theorem 4.2. Let $\mathcal{A} \in \mathbb{R}^{[m, n]}$ be a weakly symmetric nonnegative tensor. Then

$$
\rho(\mathcal{A}) \leq \Theta_{\max }(\mathcal{A})=\max \left\{\Theta_{1}(\mathcal{A}), \Theta_{2}(\mathcal{A})\right\}
$$

where $\Theta_{1}(\mathcal{A})=\max _{i \in N}\left\{R_{i}(\mathcal{A})-P_{i}^{i}(\mathcal{A})\right\}$ and

$$
\Theta_{2}(\mathcal{A})=\max _{i, j \in N, i \neq j} \min \left\{\frac{1}{2}\left(R_{i}(\mathcal{A})-P_{i}^{i}(\mathcal{A})+P_{j}^{i}(\mathcal{A})+\Upsilon_{i, j}^{\frac{1}{2}}(\mathcal{A})\right), R_{i}(\mathcal{A})\right\},
$$

with $\Upsilon_{i, j}(\mathcal{A})=\left(R_{i}(\mathcal{A})-P_{i}^{i}(\mathcal{A})-P_{j}^{i}(\mathcal{A})\right)^{2}+4 P_{i}^{i}(\mathcal{A})\left(R_{j}(\mathcal{A})-P_{j}^{i}(\mathcal{A})\right)$.

Proof. Since $\mathcal{A}$ is a weakly symmetric nonnegative tensor, we see that $\rho(\mathcal{A})$ is the largest $Z$-eigenvalue of $\mathcal{A}$ by virtue of Lemma 4.1 then it follows from Theorem 2.1 that

$$
\rho(\mathcal{A}) \in \mathcal{B}(\mathcal{A})=\left(\bigcup_{i \in N} \mathcal{B}_{i}^{1}(\mathcal{A})\right) \bigcup\left(\bigcup_{i, j \in N, i \neq j}\left(\mathcal{B}_{i, j}^{2}(\mathcal{A}) \bigcap \mathcal{K}_{i}(\mathcal{A})\right)\right) .
$$

If $\rho(\mathcal{A}) \in \bigcup_{i \in N} \mathcal{B}_{i}^{1}(\mathcal{A})$, then there exists $i_{0} \in N$ such that $\rho(\mathcal{A}) \leq R_{i_{0}}(\mathcal{A})-P_{i_{0}}^{i_{0}}(\mathcal{A})$, which yields that

$$
\rho(\mathcal{A}) \leq R_{i_{0}}(\mathcal{A})-P_{i_{0}}^{i_{0}}(\mathcal{A}) \leq \max _{i \in N}\left\{R_{i}(\mathcal{A})-P_{i}^{i}(\mathcal{A})\right\}
$$

For the case that $\rho(\mathcal{A}) \in \bigcup_{i, j \in N, i \neq j}\left(\mathcal{B}_{i, j}^{2}(\mathcal{A}) \cap \mathcal{K}_{i}(\mathcal{A})\right)$, there exist $p, q \in N$ and $p \neq q$ such that

$$
\rho(\mathcal{A}) \leq R_{p}(\mathcal{A})
$$

and

$$
\left(\rho(\mathcal{A})-\left[R_{p}(\mathcal{A})-P_{p}^{p}(\mathcal{A})\right]\right)\left(\rho(\mathcal{A})-P_{q}^{p}(\mathcal{A})\right) \leq P_{p}^{p}(\mathcal{A})\left(R_{q}(\mathcal{A})-P_{q}^{p}(\mathcal{A})\right) .
$$

Solving the quadratic inequality 4.3 gives

$$
\rho(\mathcal{A}) \leq \frac{1}{2}\left\{R_{p}(\mathcal{A})-P_{p}^{p}(\mathcal{A})+P_{q}^{p}(\mathcal{A})+\Upsilon_{p, q}^{\frac{1}{2}}(\mathcal{A})\right\}
$$

where $\Upsilon_{p, q}(\mathcal{A})=\left(R_{p}(\mathcal{A})-P_{p}^{p}(\mathcal{A})-P_{q}^{p}(\mathcal{A})\right)^{2}+4 P_{p}^{p}(\mathcal{A})\left(R_{q}(\mathcal{A})-P_{q}^{p}(\mathcal{A})\right)$.

Combining (4.2) and (4.4) gives

$$
\begin{aligned}
\rho(\mathcal{A}) & \leq \min \left\{\frac{1}{2}\left(R_{p}(\mathcal{A})-P_{p}^{p}(\mathcal{A})+P_{q}^{p}(\mathcal{A})+\Upsilon_{p, q}^{\frac{1}{2}}(\mathcal{A})\right), R_{p}(\mathcal{A})\right\} \\
& \leq \max _{i, j \in N, i \neq j} \min \left\{\frac{1}{2}\left(R_{i}(\mathcal{A})-P_{i}^{i}(\mathcal{A})+P_{j}^{i}(\mathcal{A})+\Upsilon_{i, j}^{\frac{1}{2}}(\mathcal{A})\right), R_{i}(\mathcal{A})\right\} .
\end{aligned}
$$

The conclusion follows from inequalities 4.1 and 4.5).

Remark 4.3. Due to the fact that the upper bounds for $\rho(\mathcal{A})$ in Theorems 4.6 and 4.7 in 37] are deduced from the $Z$-eigenvalue localization sets $\mathcal{M}(\mathcal{A})$ and $\mathcal{N}(\mathcal{A})$, respectively, and that in Theorem 4.2 is derived from the $Z$-eigenvalue localization set $\mathcal{B}(\mathcal{A})$, and $\mathcal{B}(\mathcal{A}) \subseteq \mathcal{M}(\mathcal{A}) \subseteq \mathcal{K}(\mathcal{A}), \mathcal{B}(\mathcal{A}) \subseteq \mathcal{N}(\mathcal{A}) \subseteq \mathcal{K}(\mathcal{A})$, we can prove that $\Theta_{\max }(\mathcal{A}) \leq \bar{v} \leq \max _{i \in N} R_{i}(\mathcal{A})$ and $\Theta_{\max }(\mathcal{A}) \leq \bar{w} \leq \max _{i \in N} R_{i}(\mathcal{A})$, where $\bar{v}$ and $\bar{w}$ are defined as in Theorems 4.6 and 4.7 in [37, respectively. 
We have showed that our bound in Theorem 4.2 is sharper than some existing ones. Now we take an example to show the efficiency of the new upper bound.

Example 4.4 ([11, 24, 37, 5] $)$. Consider the tensor $\mathcal{A}=\left(a_{i j k l}\right) \in \mathbb{R}^{[4,2]}$ with entries defined as follows:

$$
a_{1111}=\frac{1}{2}, a_{2222}=3, \text { and } a_{i j k l}=\frac{1}{3} \text { elsewhere. }
$$

We compute the bounds for $\rho(\mathcal{A})$ in Table 5 . From this table, it can be found that the upper bound in Theorem 4.2 outperforms the ones in Corollary 4.5 of [36], Theorem 2.7 of [11], Theorem 3.3 of [24, and Theorems 4.6-4.7 of [37.

TABLE 5. Some upper bounds for the $Z$-spectral radius of $\mathcal{A}$.

\begin{tabular}{ll}
\hline Corollary 4.5 of [36] & $\rho(\mathcal{A}) \leq 5.3333$ \\
\hline Theorem 2.7 of [11] & $\rho(\mathcal{A}) \leq 5.2846$ \\
\hline Theorem 3.3 of $[24]$ & $\rho(\mathcal{A}) \leq 5.1935$ \\
\hline Theorem 4.6 of [37] & $\rho(\mathcal{A}) \leq 5.1822$ \\
\hline Theorem 4.7 of [37] & $\rho(\mathcal{A}) \leq 5.1822$ \\
\hline Theorem 4.2 & $\rho(\mathcal{A}) \leq 5.1667$ \\
\hline
\end{tabular}

Theorem 4.5. Let $\mathcal{A} \in \mathbb{R}^{[m, n]}$ be a weakly symmetric nonnegative tensor with $m \geq 2$ and $R_{i}(\mathcal{A})>0$. Then

$$
\rho(\mathcal{A}) \leq \max _{a_{i_{1} \ldots i_{m}} \neq 0}\left(\prod_{l=1}^{m} R_{i_{l}}(\mathcal{A})\right)^{\frac{1}{m}} .
$$

Proof. Because $\mathcal{A}$ is a weakly symmetric nonnegative tensor, $\rho(\mathcal{A})$ is the largest $Z$-eigenvalue of $\mathcal{A}$; then it follows from Theorem 2.5 that

$$
\rho(\mathcal{A}) \in \mathcal{F}(\mathcal{A})=\bigcup_{a_{i_{1} i_{2} \ldots i_{m}} \neq 0}\left\{z \in \mathbb{C}:|z| \leq\left(\prod_{l=1}^{m} R_{i_{l}}(\mathcal{A})\right)^{\frac{1}{m}}\right\} .
$$

Since $R_{i}(\mathcal{A})>0$, there exists $a_{t_{1} t_{2} \ldots t_{m}} \neq 0$ such that

$$
\rho(\mathcal{A}) \leq\left(\prod_{l=1}^{m} R_{t_{l}}(\mathcal{A})\right)^{\frac{1}{m}}
$$

which means that

$$
\rho(\mathcal{A}) \leq \max _{a_{i_{1} \ldots i_{m}} \neq 0}\left(\prod_{l=1}^{m} R_{i_{l}}(\mathcal{A})\right)^{\frac{1}{m}} .
$$

This completes our proof. 
Remark 4.6. Similar to the discussions in Remark 4.3, we conclude that the upper bound in Theorem 4.5 is better than that in Corollary 3.5 of [36].

Let us show the advantage of the new upper bound in Theorem 4.5 over the one in Corollary 3.5 of 36 by a simple example as follows.

Example 4.7. Consider the weakly symmetric nonnegative tensor

$$
\mathcal{A}=[A(1,:,:), A(2,:,:), A(3,:,:)] \in \mathbb{R}^{[3,3]},
$$

where

$$
A(1,:,:)=\left(\begin{array}{ccc}
0 & 3 & 0 \\
3 & 1 & 2 \\
0 & 2 & 1.5
\end{array}\right), A(2,:,:)=\left(\begin{array}{ccc}
3 & 1 & 2 \\
1 & 0 & 2.5 \\
2 & 2.5 & 1
\end{array}\right), A(3,:,:)=\left(\begin{array}{ccc}
0 & 2 & 1.5 \\
2 & 2.5 & 1 \\
1.5 & 1 & 0
\end{array}\right) .
$$

We compare the upper bound exhibited in Theorem 4.5 with that in Corollary 4.5 of [36] in Table 6. From the observations in this table, it can be clearly seen that the upper bound in Theorem 4.5 is tighter than that in Corollary 4.5 of [36], which is in accordance with the conclusions in Theorem 2.6 and Remark 4.6.

TABLE 6. Upper bounds for the $Z$-spectral radius of $\mathcal{A}$.

\begin{tabular}{lll}
\hline Corollary 4.5 of $[36]$ & $\rho(\mathcal{A}) \leq 15$ \\
\hline Theorem 4.5 & & $\rho(\mathcal{A}) \leq 14.1155$ \\
\hline
\end{tabular}

Remark 4.8. We have recently developed a new $S$-type eigenvalue localization set for tensors in [14]. The differences between both papers are as follows:

- The kinds of the eigenvalue localization sets of these two papers are different. Both papers introduce some eigenvalue localization sets for tensors. While the main aim of the present paper is to establish some new $Z$-eigenvalue (see Definition 1.2) localization sets for tensors, in [14 we derive a new $S$-type eigenvalue (see Definition 1.1) localization set for tensors.

- There is a difference between the techniques used in these two papers. Theorem 3.1 of 14 is derived by breaking $N$ into disjoint subsets $S$ and its complement, and the eigenvalue localization set proposed in 14 relies on the choice of $S$. But the $Z$-eigenvalue localization sets developed in the present paper do not contain the set $S$.

- The applications of eigenvalue localization sets in the two papers are different. One of the applications of the eigenvalue localization set in [14 is obtaining new sufficient conditions for positive (semi-)definiteness of tensors, which involve the $H$-eigenvalues of tensors, but this application can not be established by the $Z$-eigenvalue localization set in the present paper. 


\section{Concluding REMARKS}

To localize all $Z$-eigenvalues of a tensor much sharper compared with some known ones, some new $Z$-eigenvalue localization sets for tensors are derived in this paper, which are proved to be more precise than the ones in [37, and we also study the effects of orthonormal transformations for the proposed sets. Based on the new sets, we establish some new upper bounds for the $Z$-spectral radius of weakly symmetric nonnegative tensors. These bounds are tighter than those proposed by Wang et al. 37] in theory. Some numerical results worked out in this paper verify that the proposed results are superior to some existing ones.

Besides, we should mention that there exist some problems to be studied in the future:

- Study the new $Z$-eigenvalue localization set which is sharper than the one in Lemma 1.7 in theory.

- Research the relationship between the set $\mathcal{B}(\mathcal{A})$ and the set $\mathcal{F}(\mathcal{A})$.

- Choose the optimal orthogonal matrix $P$ such that $\mathcal{B}(\mathcal{A})$ and $\mathcal{F}(\mathcal{A})$ are best under orthonormal transformations.

\section{ACKNOWLEDGMENTS}

We would like to express our sincere thanks to the editor and the anonymous reviewers for their valuable suggestions and constructive comments which greatly improved the presentation of this paper.

\section{REFERENCES}

[1] C. J. Bu, X. Q. Jin, H. F. Li and C. L. Deng, Brauer-type eigenvalue inclusion sets and the spectral radius of tensors, Linear Algebra Appl. 512 (2017), 234-248. MR 3567524

[2] C. J. Bu, Y. P. Wei, L. Z. Sun and J. Zhou, Brualdi-type eigenvalue inclusion sets of tensors, Linear Algebra Appl. 480 (2015), 168-175. MR 3348519.

[3] K. C. Chang, K. J. Pearson and T. Zhang, On eigenvalue problems of real symmetric tensors, J. Math. Anal. Appl. 350 (2009), no. 1, 416-422. MR 2476927.

[4] K.-C. Chang, K. J. Pearson and T. Zhang, Primitivity, the convergence of the NQZ method, and the largest eigenvalue for nonnegative tensors, SIAM J. Matrix Anal. Appl. 32 (2011), no. 3, 806-819. MR 2837580

[5] K. C. Chang, K. J. Pearson and T. Zhang, Some variational principles for Z-eigenvalues of nonnegative tensors, Linear Algebra Appl. 438 (2013), no. 11, 4166-4182. MR 3034523

[6] K. C. Chang and T. Zhang, On the uniqueness and non-uniqueness of the positive $Z$ eigenvector for transition probability tensors, J. Math. Anal. Appl. 408 (2013), no. 2, 525540. MR 3085049 .

[7] J. Cooper and A. Dutle, Spectra of uniform hypergraphs, Linear Algebra Appl. 436 (2012), no. 9, 3268-3292. MR 2900714

[8] W. Y. Ding, L. Q. Qi and Y. M. Wei, $\mathscr{M}$-tensors and nonsingular $\mathscr{M}$-tensors, Linear Algebra Appl. 439 (2013), no. 10, 3264-3278. MR 3116429

[9] W. Y. Ding and Y. M. Wei, Generalized tensor eigenvalue problems, SIAM J. Matrix Anal. Appl. 36 (2015), no. 3, 1073-1099. MR 3376129.

[10] J. He and T. Z. Huang, Inequalities for M-tensors, J. Inequal. Appl. 2014 (2014), 114. MR 3346818

[11] J. He and T. Z. Huang, Upper bound for the largest Z-eigenvalue of positive tensors, Appl. Math. Lett. 38 (2014), 110-114. MR 3258212. 
[12] S. L. Hu, Z. H. Huang and L. Q. Qi, Strictly nonnegative tensors and nonnegative tensor partition, Sci. China Math. 57 (2014), no. 1, 181-195. MR 3146525.

[13] Z.-G. Huang, L.-G. Wang, Z. Xu and J.-J. Cui, A new S-type eigenvalue inclusion set for tensors and its applications, J. Inequal. Appl. 2016, Paper No. 254, 19 pp. MR 3561908

[14] Z. G. Huang, L. G. Wang, Z. Xu and J. J. Cui, A new S-type eigenvalue localization set for tensors and its applications, preprint, 2016. arXiv:1602.07568 [math.CO].

[15] E. Kofidis and P. A. Regalia, On the best rank-1 approximation of higher-order supersymmetric tensors, SIAM J. Matrix Anal. Appl. 23 (2001/02), no. 3, 863-884. MR 1896822

[16] C. Q. Li, Z. Chen and Y. T. Li, A new eigenvalue inclusion set for tensors and its applications, Linear Algebra Appl. 481 (2015), 36-53. MR 3349643

[17] C. Q. Li, A. Q. Jiao and Y. T. Li, An S-type eigenvalue localization set for tensors, Linear Algebra Appl. 493 (2016), 469-483. MR 3452751

[18] C. Q. Li and Y. T. Li, An eigenvalue localization set for tensors with applications to determine the positive (semi-)definiteness of tensors, Linear Multilinear Algebra 64 (2016), no. 4, 587601. MR 3479020

[19] C. Q. Li and Y. T. Li, Relationships between Brauer-type eigenvalue inclusion sets and a Brualdi-type eigenvalue inclusion set for tensors, Linear Algebra Appl. 496 (2016), 71-80. MR 3464062

[20] C. Q. Li, Y. T. Li and X. Kong, New eigenvalue inclusion sets for tensors, Numer. Linear Algebra Appl. 21 (2014), no. 1, 39-50. MR 3150608

[21] C. Q. Li, F. Wang, J. X. Zhao, Y. Zhu and Y. T. Li, Criterions for the positive definiteness of real supersymmetric tensors, J. Comput. Appl. Math. 255 (2014), 1-14. MR 3093400

[22] C. Q. Li, J. J. Zhou and Y. T. Li, A new Brauer-type eigenvalue localization set for tensors, Linear Multilinear Algebra 64 (2016), no. 4, 727-736. MR 3479029.

[23] G. Y. Li, L. Q. Qi and G. H. Yu, The Z-eigenvalues of a symmetric tensor and its application to spectral hypergraph theory, Numer. Linear Algebra Appl. 20 (2013), no. 6, 1001-1029. MR 3141890

[24] W. Li, D. D. Liu and S. W. Vong, Z-eigenpair bounds for an irreducible nonnegative tensor, Linear Algebra Appl. 483 (2015), 182-199. MR 3378896

[25] W. Li and M. K. Ng, On the limiting probability distribution of a transition probability tensor, Linear Multilinear Algebra 62 (2014), no. 3, 362-385. MR 3175419

[26] W. Li and M. K. Ng, Some bounds for the spectral radius of nonnegative tensors, Numer. Math. 130 (2015), no. 2, 315-335. MR 3343927.

[27] L. H. Lim, Singular values and eigenvalues of tensors: a variational approach, in: Proc. 1st IEEE International Workshop on Computational Advances of Multi-Sensor Adaptive Processing, 2005, 129-132.

[28] M. Ng, L. Q. Qi and G. L. Zhou, Finding the largest eigenvalue of a nonnegative tensor, SIAM J. Matrix Anal. Appl. 31 (2009), no. 3, 1090-1099. MR 2538668

[29] L. Q. Qi, Eigenvalues of a real supersymmetric tensor, J. Symbolic Comput. 40 (2005), no. 6, 1302-1324. MR 2178089

[30] L. Q. Qi, Eigenvalues and invariants of tensors, J. Math. Anal. Appl. 325 (2007), no. 2, 1363-1377. MR 2270090.

[31] L. Q. Qi, Symmetric nonnegative tensors and copositive tensors, Linear Algebra Appl. 439 (2013), no. 1, 228-238. MR 3045233

[32] L. Q. Qi and Z. Y. Luo, Tensor analysis: spectral theory and special tensors, Society for Industrial and Applied Mathematics, Philadelphia, 2017. MR 3660696.

[33] L. Q. Qi, F. Wang and Y. Wang, Z-eigenvalue methods for a global polynomial optimization problem, Math. Program. 118 (2009), no. 2, Ser. A, 301-316. MR 2470793.

[34] L. Q. Qi, G. H. Yu and E. X. Wu, Higher order positive semidefinite diffusion tensor imaging, SIAM J. Imaging Sci. 3 (2010), no. 3, 416-433. MR 2679434.

[35] C. L. Sang, A new Brauer-type Z-eigenvalue inclusion set for tensors, Numer. Algorithms 80 (2019), no. 3, 781-794. MR 3923621 
[36] Y. S. Song and L. Q. Qi, Spectral properties of positively homogeneous operators induced by higher order tensors, SIAM J. Matrix Anal. Appl. 34 (2013), no. 4, 1581-1595. MR 3131483.

[37] G. Wang, G. L. Zhou and L. Caccetta, Z-eigenvalue inclusion theorem for tensors, Discrete Contin. Dyn. Syst. Ser. B 22 (2017), no. 1, 187-198. MR 3583465

[38] G. Wang, G. L. Zhou and L. Caccetta, Sharp Brauer-type eigenvalue inclusion theorems for tensors, Pac. J. Optim. 14 (2018), no. 2, 227-244.

[39] X.-Z. Wang and Y. M. Wei, Bounds for eigenvalues of nonsingular $\mathcal{H}$-tensor, Electronic J. Linear Algebra 29 (2015), 3-16. MR 3414581

[40] Q. Z. Yang and Y. N. Yang, Further results for Perron-Frobenius theorem for nonnegative tensors II, SIAM J. Matrix Anal. Appl. 32 (2011), no. 4, 1236-1250. MR 2854611.

[41] Y. N. Wang and G. Wang, Two $S$-type $Z$-eigenvalue inclusion sets for tensors, J. Inequal. Appl. 2017, Paper no. 152, 12 pp. MR 3666453

[42] Y. N. Yang and Q. Z. Yang, Further results for Perron-Frobenius theorem for nonnegative tensors, SIAM J. Matrix Anal. Appl. 31 (2010), no. 5, 2517-2530. MR 2685169.

[43] T. Zhang and G. H. Golub, Rank-one approximation to high order tensors, SIAM J. Matrix Anal. Appl. 23 (2001), no. 2, 534-550. MR 1871328

\author{
Zhengge Huang \\ Department of Applied Mathematics, School of Science, Northwestern Polytechnical University, \\ Xi'an, Shaanxi 710072, P. R. China \\ ZhenggeHuang@mail.nwpu.edu.cn \\ Ligong Wang ${ }^{凶}$ \\ Department of Applied Mathematics, School of Science, Northwestern Polytechnical University, \\ Xi'an, Shaanxi 710072, P. R. China \\ lgwang@nwpu.edu.cn \\ Zhong $X u$ \\ Department of Applied Mathematics, School of Science, Northwestern Polytechnical University, \\ Xi'an, Shaanxi 710072, P. R. China \\ zhongxu@nwpu.edu.cn \\ Jingjing Cui \\ Department of Applied Mathematics, School of Science, Northwestern Polytechnical University, \\ Xi'an, Shaanxi 710072, P. R. China \\ cuijingjinghehuang@163.com
}

Received: March 9, 2018

Accepted: September 11, 2018 